\title{
CONFORMAL AND RELATED CHANGES OF METRIC ON THE PRODUCT OF TWO ALMOST CONTACT METRIC MANIFOLDS
}

\author{
D.E. Blair and J.A. OUBIÑA
}

\begin{abstract}
This paper studies conformal and related changes of the product metric on the product of two almost contact metric manifolds. It is shown that if one factor is Sasakian, the other is not, but that locally the second factor is of the type studied by Kenmotsu. The results are more generaI and given in terms of trans-Sasakian, $\alpha$-Sasakian and $\beta$-Kenmotsu structures.
\end{abstract}

\section{Introduction}

The product of an almost contact manifold $M$ and the real line $\mathbf{R}$ carries a natural almost complex structure. When this structure is integrable the almost contact structure is said to be normal. In the contact case, a normal contact metric manifold is called a Sasakian manifold. Moreover the product of two almost contact manifolds also carries a natural almost complex structure whose integrability is equivalent to the normality of both almost contact structures [8]. However if one takes $M$ to be an almost contact metric manifold and supposes that the product metric $G$ on $M \times \mathbf{R}$ is Kaehlerian, then the structure on $M$ is cosymplectic [4] and not Sasakian. On the other hand the second author pointed out in [9] that if the conformally related metric $e^{2 t} G, t$ being the coordinate on $\mathbf{R}$, is Kaehlerian, then $M$ is Sasakian and conversely. In [2] Capursi showed that for the product of two almost contact metric manifolds, the product metric is Kaehlerian if and only if both factors are cosymplectic. This raises the still open question: What kind of change of the product metric will make both factors Sasakian? Here we study conformal and related changes of the product metric and show that if one factor is Sasakian the other is not, but that locally the second factor is of the type studied by Kenmotsu [6]. This structure will be described in section 3 and in section 4 we shall consider trans-Sasakian structures [9], which will be used for our main results in section 5 . 


\section{Almost contact manifolds}

An almost contact manifold is an odd-dimensional $C^{\infty}$ manifold whose structural group can be reduced to $U(n) \times 1$. This is equivalent to the existence of a tensor field $\phi$ of type $(1,1)$, a vector field $\xi$ and a 1 -form $\eta$ satisfying $\phi^{2}=-I+\eta \otimes \xi$ and $\eta(\xi)=1$. From these conditions one can deduce that $\phi \xi=0$ and $\eta \circ \phi=0$. A Riemannian metric $g$ is compatible with these structure tensors if

$$
g(\phi X, \phi Y)=g(X, Y)-\eta(X) \eta(Y)
$$

and we refer to an almost contact metric structure $(\phi, \xi, \eta, g)$. Note also that $\eta(X)=g(X, \xi)$. For a general reference to the ideas of this section see [1].

Let $M$ be an almost contact manifold and define an almost complex structure $J$ on $M \times \mathrm{R}$ by

$$
J\left(X, f \frac{d}{d t}\right)=\left(\phi X-f \xi, \eta(X) \frac{d}{d t}\right)
$$

An almost contact structure is said to be normal if $J$ is integrable. If in addition to the conditions for an almost contact metric structure, we have $d \eta(X, Y)=$ $g(X, \phi Y)$, the structure is a contact metric structure, in particular if $\operatorname{dim} M=$ $2 n+1, \eta \Lambda(d \eta)^{n} \neq 0$. Then a Sasakian manifold is a normal contact metric manifold. It is well known that the Sasakian condition may be expressed as an almost contact metric structure satisfying

$$
(\nabla X \phi) Y=g(X, Y) \xi-\eta(Y) X
$$

again see e.g. [1].

More generally one has the notion of an $\alpha$-Sasakian structure [5] which may be defined by the requirement

$$
(\nabla X \phi) Y=\alpha(g(X, Y) \xi-\eta(Y) X)
$$

where $\alpha$ is a non-zero constant. Setting $Y=\xi$ in this formula, one readily obtains

$$
\nabla x \xi=-\alpha \phi X
$$

\section{Kenmotsu manifolds}

In [10] Tanno classified connected almost contact metric manifolds whose automorphism groups have the maximum dimension. For such a manifold $M$, the sectional curvature of plane sections containing $\xi$ is a constant, say $c$. If $c>0, M$ is a homogeneous Sasakian manifold of constant $\phi$-sectional curvature. If $c=0, M$ is the product of a line or circle with a. Kaehler manifold of constant holomorphic curvature. If $c<0, M$ is a warped product space $\mathbf{R} \times{ }_{f} \mathbf{C}^{n}$. In [6] Kenmotsu abstracted the differential geometric properties of the third case. In particular the almost contact metric structure in this case satisfies

$$
(\nabla X \phi) Y=g(\phi X, Y) \xi-\eta(Y) \phi X
$$

and an almost contact metric manifold satisfying this condition is called a Kenmotsu manifold $[5,6]$. Kenmotsu proved in particular the following result. 
Theorem. (Kenmotsu [6]) Let $M$ be a Kenmotsu manifold. Then for any point peM, there is a neighborhood $U$ of $p$ which is a warped product $(-\varepsilon, \varepsilon) \times{ }_{f} V$ where $f(t)=c e^{t}$ on the interval $(-\varepsilon, \varepsilon)$ and $V$ is a Kaehler manifold.

Again one has the more general notion of a $\beta$-Kenmotsu structure [5] which may be defined by

$$
(\nabla X \phi) Y=\beta(g(\phi X, Y) \xi-\eta(Y) \phi X)
$$

where $\beta$ is a non-zero constant. From the condition one may readily deduce that

$$
\nabla x \xi=\beta(X-\eta(X) \xi)
$$

\section{Trans-Sasakian manifolds}

In the classification of Gray and Hervella [3] of almost Hermitian manifolds there appears a class, $W_{4}$, of Hermitian manifolds which are closely related to locally conformally Kaehler manifolds. An almost contact metric structure $(\phi, \xi, \eta, g)$ on $M$ is trans-Sasakian $[9]$ if $(M \times \mathbf{R}, J, G)$ belongs to the class $W_{4}$, where $J$ is the almost complex structure on $M \times \mathbf{R}$ defined by (2.1) and $G$ is the product metric on $M \times \mathbf{R}$. This may be expressed by the condition

$$
(\nabla X \phi) Y=\alpha(g(X, Y) \xi-\eta(Y) X)+\beta(g(\phi X, Y) \xi-\eta(Y) \phi X)
$$

for functions $\alpha$ and $\beta$ on $M$, and we shall say that the trans-Sasakian structure is of type $(\alpha, \beta)$; in particular, it is normal and it generalizes both $\alpha$-Sasakian and $\beta$-Kenmotsu structures. From the formula one easily obtains

$$
\begin{gathered}
\nabla x \xi=-\alpha \phi X+\beta(X-\eta(X) \xi), \\
(\nabla x \eta)(Y)=-\alpha g(\phi X, Y)+\beta(g(X, Y)-\eta(X) \eta(Y)), \\
(\nabla \times \Phi)(Y, Z)=\alpha(g(X, Z) \eta(Y)-g(X, Y) \eta(Z))-\beta(g(X, \phi Z) \eta(Y)-g(X, \phi Y) \eta(Z))
\end{gathered}
$$

where $\Phi$ is the fundamental 2 -form of the structure, given by $\Phi(X, Y)=$ $g(X, \phi Y)$. Hence

$$
(\nabla X \Phi)(X, \xi)=-\alpha,(\nabla X \eta)(X)=\beta
$$

for $X$ orthogonal to $\xi$, and $g(X, X)=1$. Then

$$
\delta \Phi(\xi)=2 n \alpha, \delta \eta=-2 n \beta
$$

where $\delta$ is the codifferential of $g$ and $\operatorname{dim} M=2 n+1$. Moreover $d \eta=\alpha \Phi$. If $\alpha$ is a non-zero constant, $\Phi$ is closed and one has (cf. [1], p.53)

$$
\begin{aligned}
g((\nabla \times \phi) Y, Z) & =d \eta(\phi Y, X) \eta(Z)-d \eta(\phi Z, X) \eta(Y) \\
& =\alpha g(X, Y) \eta(Z)-\alpha g(X, Z) \eta(Y)
\end{aligned}
$$


Then

$$
(\nabla \times \phi) Y=\alpha(g(X, Y) \xi-\eta(Y) X)
$$

Thus $\beta=0$ and therefore a trans-Sasakian structure of type $(\alpha, \beta)$ with $\alpha$ a non-zero constant is $\alpha$-Sasakian.

Example. Let $(x, y, z)$ be cartesian coordinates on $\mathbf{R}^{3}$ and put

$$
\begin{gathered}
\xi=\frac{\partial}{\partial z}, \eta=d z-y d x \\
\phi=\left(\begin{array}{rrr}
0 & -1 & 0 \\
1 & 0 & 0 \\
0 & -y & 0
\end{array}\right), g=\left(\begin{array}{ccr}
e^{z}+y^{2} & 0 & -y \\
0 & e^{z} & 0 \\
-y & 0 & 1
\end{array}\right)
\end{gathered}
$$

Then $\delta \Phi(\xi)=-\frac{1}{e^{z}}, \delta \eta=-1$ and $(\phi, \xi, \eta, g)$ is a trans-Sasakian structure on $\mathbf{R}^{3}$ of type $\left(-\frac{1}{2 e^{z}}, \frac{1}{2}\right)$.

The relation between trans-Sasakian, $\alpha$-Sasakian and $\beta$-Kenmotsu structures was recently discussed by Marrero [7].

Proposition 4.1. (Marrero [7]) Let $M$ be a 3-dimensional Sasakian manifold with structure tensors $(\phi, \xi, \eta, g), f>0$ a non-constant function on $M$ and $\bar{g}=f g+(1-f) \eta \otimes \eta$. Then $(\phi, \xi, \eta, \bar{g})$ is a trans-Sasaksan structure of type $\left(\frac{1}{f}, \frac{1}{2} \xi(\ln f)\right)$.

Proposition 4.2. (Marrero [7]) A trans-Sasakian manifold of dimension $\geq 5$ is either $\alpha$-Sasakian, $\beta$-Kenmotsu or cosymplectic.

\section{A Study of $M_{1} \times M_{2}$}

Let $M_{1}$ and $M_{2}$ be almost contact metric manifolds with structure tensors $\left(\phi_{i}, \xi_{i}, \eta_{i}, g_{i}\right), i=1,2$. Define an almost complex structure $J$ on $M_{1} \times M_{2}$ by

$$
J\left(X_{1}, X_{2}\right)=\left(\phi_{1} X_{1}-e^{-2 \mu} \eta_{2}\left(X_{2}\right) \xi_{1}, \phi_{2} X_{2}+e^{2 \mu} \eta_{1}\left(X_{1}\right) \xi_{2}\right)
$$

where $\mu$ is a function on $M_{1} \times M_{2}$. That $J^{2}=-I$ is easily checked. Let $\tilde{g}$ be the Riemannian metric on $M_{1} \times M_{2}$ defined by

$$
\tilde{g}\left(\left(X_{1}, X_{2}\right),\left(Y_{1}, Y_{2}\right)\right)=e^{2 \rho} g_{1}\left(X_{1}, Y_{1}\right)+e^{2 \tau} g_{2}\left(X_{2}, Y_{2}\right)
$$

where $\rho$ and $\tau$ are functions on $M_{1} \times M_{2}$. Then $\tilde{g}$ is Hermitian with respect to $J$, i.e.

$$
\tilde{g}\left(J\left(X_{1}, X_{2}\right), J\left(Y_{1}, Y_{2}\right)\right)=\tilde{g}\left(\left(X_{1}, X_{2}\right),\left(Y_{1}, Y_{2}\right)\right)
$$

if and only if

$$
\mu=\frac{1}{2}(\rho-\tau)
$$


Let $\nabla^{1}, \nabla^{2}$ and $\tilde{\nabla}$ denote the Riemannian connections of $g_{1}, g_{2}$ and $\tilde{g}$ respectively. Now taking $X_{1}$ and $Y_{1}$ as vector fields tangent to $M_{1}$ and independent of $M_{2}$ and similarly for $X_{2}$ and $Y_{2}$ we give the connection $\tilde{\nabla}$ explicitly:

$\tilde{\nabla}_{\left(X_{1}, 0\right)}\left(Y_{1}, 0\right)=$

$\left(\nabla_{X_{1}}^{\mathrm{t}} Y_{1}+\left(X_{1} \rho\right) Y_{1}+\left(Y_{1} \rho\right) X_{1}-g_{1}\left(X_{1}, Y_{1}\right) \operatorname{grad}^{1} \rho,-e^{2(\rho-r)} g_{1}\left(X_{1}, Y_{1}\right) \operatorname{grad}^{2} \rho\right)$

$\tilde{\nabla}_{\left(0, X_{2}\right)}\left(0, Y_{2}\right)=$

$\left(-e^{2(\tau-\rho)} g_{2}\left(X_{2}, Y_{2}\right) \operatorname{grad}^{1} \tau, \nabla_{X_{2}}^{2} Y_{2}+\left(X_{2} \tau\right) Y_{2}+\left(Y_{2} \tau\right) X_{2}-g_{2}\left(X_{2}, Y_{2}\right) \operatorname{grad}^{2} \tau\right)$

$$
\begin{aligned}
& \tilde{\nabla}_{\left(X_{1}, 0\right)}\left(0, Y_{2}\right)=\left(\left(Y_{2} \rho\right) X_{1},\left(X_{1} \tau\right) Y_{2}\right) \\
& \tilde{\nabla}_{\left(0, X_{2}\right)}\left(Y_{1}, 0\right)=\left(\left(X_{2} \rho\right) Y_{1},\left(Y_{1} \tau\right) X_{2}\right)
\end{aligned}
$$

Now taking $\mu=\frac{1}{2}(\rho-\tau)$, we compute the covariant derivative of $J$.

$\left(\tilde{\nabla}_{\left(X_{1}, 0\right)} J\right)\left(Y_{1}, 0\right)=\left(\left(\nabla^{1} X_{1} \phi_{1}\right) Y_{1}+\left(\phi_{1} Y_{1} \rho\right) X_{1}\right.$

$-\left(Y_{1} \rho\right) \phi_{1} X_{1}-g_{1}\left(X_{1}, \phi_{1} Y_{1}\right) \operatorname{grad}^{1} \rho$

$+g_{1}\left(X_{1}, Y_{1}\right) \phi_{1} \operatorname{grad}^{1} \rho+e^{\rho-\tau}\left(\xi_{2} \rho\right) \eta_{1}\left(Y_{1}\right) X_{1}$

$-e^{\rho-\tau}\left(\xi_{2} \rho\right) g_{1}\left(X_{1}, Y_{1}\right) \xi_{1}$,

$e^{\rho-\tau}\left(\nabla_{X_{1}}^{1} \eta_{1}\right)\left(Y_{2}\right) \xi_{2}$

$-e^{\rho-\tau}\left(Y_{1} \rho\right) \eta_{1}\left(X_{1}\right) \xi_{2}+e^{\rho-\tau}\left(\xi_{1} \rho\right) g_{1}\left(X_{1}, Y_{1}\right) \xi_{2}$

$\left.-e^{2(\rho-\tau)} g_{1}\left(X_{1}, \phi_{1} Y_{2}\right) \operatorname{grad}^{2} \rho+e^{2(\rho-\tau)} g_{1}\left(X_{1}, Y_{1}\right) \phi_{2} \operatorname{grad}^{2} \rho\right)$

$\left(\tilde{\nabla}_{\left(0, X_{2}\right)} J\right)\left(0, Y_{2}\right)=\left(-e^{r-\rho}\left(\nabla_{X_{2}}^{2} \eta_{2}\right)\left(Y_{2}\right) \xi_{1}+e^{\tau-\rho}\left(Y_{2} \tau\right) \eta_{2}\left(X_{2}\right) \xi_{1}\right.$

$-e^{\tau-\rho}\left(\xi_{2} \tau\right) g_{2}\left(X_{2}, Y_{2}\right) \xi_{1}-e^{2(+-\rho)} g_{2}\left(X_{2}, \phi_{2} Y_{2}\right) \operatorname{grad}^{1} \tau$

$+e^{z(r-\rho)} g_{2}\left(X_{2}, Y_{2}\right) \phi_{2} \operatorname{grad}^{1} r$,

$\left(\nabla_{X_{2}}^{2} \phi_{2}\right) Y_{2}+\left(\phi_{2} Y_{2} \tau\right) X_{2}$

$-\left(Y_{2} \tau\right) \phi_{2} X_{2}-g_{2}\left(X_{2}, \phi_{2} Y_{2}\right) \operatorname{grad}^{2} \tau+g_{2}\left(X_{2}, Y_{2}\right) \phi_{2} \operatorname{grad}^{2} \tau$

$\left.-e^{\tau-\rho}\left(\xi_{1} \tau\right) \eta_{2}\left(Y_{2}\right) X_{2}+e^{\tau-\rho}\left(\xi_{1} \tau\right) g_{2}\left(X_{2}, Y_{2}\right) \xi_{2}\right)$

$\left(\tilde{\nabla}\left(X_{1}, 0\right) J\right)\left(0, Y_{2}\right)=\left(-e^{\tau-\rho_{\eta_{2}}}\left(Y_{2}\right) \nabla_{X_{1}}^{1} \xi-e^{\tau-\rho}\left(\xi_{1} \rho\right) \eta_{2}\left(Y_{2}\right) X_{1}\right.$

$+e^{r-\rho} \eta_{1}\left(X_{1}\right) \eta_{2}\left(Y_{2}\right) \operatorname{grad}^{1} \rho+\left(\phi_{2} Y_{2} \rho\right) X_{1}-\left(Y_{2} \rho\right) \phi_{1} X_{1}$, $\left.e^{\rho-\tau} \eta_{1}\left(X_{1}\right) \eta_{2}\left(Y_{2}\right) \operatorname{grad}^{2} \rho-e^{\rho-\tau}\left(Y_{2} \rho\right) \eta_{1}\left(X_{1}\right) \xi_{2}\right)$

$\left(\tilde{\nabla}_{\left(0, X_{2}\right)} J\right)\left(Y_{1}, 0\right)=\left(-e^{r-p_{\eta_{1}}}\left(Y_{\mathrm{I}}\right) \eta_{2}\left(X_{2}\right) \operatorname{grad}^{1} \tau\right.$ $+e^{r-\rho}\left(Y_{1} \tau\right) \eta_{2}\left(X_{2}\right) \xi_{1}$

$$
\begin{aligned}
& e^{\rho-\tau} \eta_{1}\left(Y_{1}\right) \nabla_{X_{3}}^{2} \xi_{2}+e^{\rho-\tau}\left(\xi_{2} \tau\right) \eta_{1}\left(Y_{I}\right) X_{2} \\
- & \left.e^{\rho-\tau} \eta_{1}\left(Y_{1}\right) \eta_{2}\left(X_{2}\right) \operatorname{grad}^{2} \tau+\left(\phi_{1} Y_{1} \tau\right) X_{2}-\left(Y_{1} \tau\right) \phi_{2} X_{2}\right)
\end{aligned}
$$


We now suppose that $\left(M_{1} \times M_{2}, J, \tilde{g}\right)$ is Kaehlerian and study the question of $M_{1}$ being trans-Sasakian. If $M_{\mathrm{I}}$ is trans-Sasakian of type $(\alpha, \beta)$, the first component of $(5.1)$ becomes

$$
\begin{aligned}
\alpha\left(g_{1}\left(X_{1}, Y_{1}\right) \xi_{1}-\eta_{1}\left(Y_{1}\right) X_{1}\right) & +\beta\left(g_{1}\left(\phi_{1} X_{1}, Y_{1}\right) \xi_{1}-\eta_{1}\left(Y_{1}\right) \phi_{1} X_{1}\right) \\
& +\left(\phi_{1} Y_{1} \rho\right) X_{1}-\left(Y_{1} \rho\right) \phi_{1} X_{1}-g_{1}\left(X_{1}, \phi_{1} Y_{1}\right) \operatorname{grad}^{1} \rho \\
& +g_{1}\left(X_{1}, Y_{1}\right) \phi_{1} \operatorname{grad}^{1} \rho+e^{\rho-\tau}\left(\xi_{2} \rho\right) \eta_{1}\left(Y_{1}\right) X_{1} \\
& -e^{\rho-\tau}\left(\xi_{2} \rho\right) g_{1}\left(X_{1}, Y_{1}\right) \xi_{1}=0
\end{aligned}
$$

Setting $X_{1}=Y_{1}$ and orthogonal to $\xi_{1}$, the $\xi_{1}$-component yields

$$
\xi_{2} \rho=\alpha e^{r-\rho}
$$

Setting $Y_{1}=\xi_{1}$ and taking $X_{1}$ orthogonal to $\xi_{1}$ we then have

$$
\xi_{1} \rho=-\beta
$$

Setting $X_{1}=\xi_{1}$ and $Y_{2}=\xi_{2}$ in the first component of (5.3) we obtain

$$
\operatorname{grad}^{1} \rho=\left(\xi_{1} \rho\right) \xi_{1}
$$

Conversely if $\operatorname{grad}^{1} \rho=-\beta \xi_{1}$ and $\xi_{2} \rho=\alpha e^{\tau-\rho}$, where $\alpha$ and $\beta$ are functions on $M_{1}$, it is easy to see that $\left(\nabla_{X_{1}}^{1} \phi_{1}\right) Y_{1}=\alpha\left(g_{1}\left(X_{1}, Y_{1}\right) \xi_{1}-\eta_{1}\left(Y_{1}\right) X_{1}\right)+$ $\beta\left(g_{1}\left(\phi_{1} X_{1}, Y_{1}\right) \xi_{1}-\eta_{1}\left(Y_{1}\right) \phi_{1} X_{1}\right)$. Note also from the second component of (5.3) we have immediately that $\operatorname{grad}^{2} \rho=\left(\xi_{2} \rho\right) \xi_{2}$. Thus we have the following proposition.

Proposition 5.1. Suppose that $\left(M_{1} \times M_{2}, J, \tilde{g}\right)$ is Kaehlerian. Then $M_{1}$ is trans-Sasakian of type $(\alpha, \beta)$ if and only if $\operatorname{grad}^{1} \rho=-\beta \xi_{1}$ and $\xi_{2} \rho=\alpha e^{\tau-\rho}$ in which case $\operatorname{grad}^{2} \rho=\alpha e^{r-\rho} \xi_{2}$.

If $\beta=0$, it follows from (5.6) and (5.7) that $\rho$ is independent of $M_{1}$ and we have the following corollary.

Corollary. Suppose that $\left(M_{1} \times M_{2}, J, \tilde{g}\right)$ is Kaehlerian and $\alpha$ is a non-zero constant. Then $M_{1}$ is $\alpha$-Sasakian if and only if grad ${ }^{2} \rho=0$ and $\xi_{2} \rho=\alpha e^{r-\rho}$.

Remark. Suppose that $M_{1}$ is $\alpha$-Sasakian and let $X_{1}$ be a local coordinate field on $M_{1}$. Then $0=X_{1} \xi_{2} \rho=\alpha e^{\tau-\rho}\left(X_{1} \tau\right)$ and hence $\tau$ is also independent of $M_{1}$. Thus we have that if $\left(M_{1} \times M_{2}, J, \tilde{g}\right)$ is Kaehlerian and $M_{1}$ is $\alpha$-Sasakian, $M_{2}$ cannot be $\alpha$-Sasakian for any constant; for then $\rho$ and $\tau$ would also be independent of $M_{2}$ and hence constant on $M_{1} \times M_{2}$. This would then give $\alpha=0$ on $M_{1}$ by (5.5), a contradiction.

Similarly to Proposition 5.1 we have the following. 
Proposition 5.2. Suppose that $\left(M_{1} \times M_{2}, J, \tilde{g}\right)$ is Kaehlerian; then $M_{2}$ is trans-Sasakian of type $(\alpha, \beta)$ if and only if $\xi_{1} \tau=-\alpha e^{\rho-\tau}$ and grad $^{2} \tau=-\beta \xi_{2}$.

Corollary. Suppose that $\left(M_{1} \times M_{2}, J, \tilde{g}\right)$ is Kaehlerian; then $M_{2}$ is $\beta \cdot$ Kenmotsu if and only if $\xi_{1} \tau=0$ and grad $d^{2} \tau=-\beta \xi_{2}$.

Now, let us consider again the almost contact metric manifolds $M_{1}$ and $M_{2}$ and the almost Hermitian manifold $\left(M_{1} \times M_{2}, J, \tilde{g}\right)$. Suppose that

$$
\begin{gathered}
\operatorname{grad}^{1} \rho=-\beta_{1} \xi_{1}, \operatorname{grad}^{2} \rho=\alpha_{1} e^{\tau-\rho} \xi_{2} \\
\operatorname{grad}^{1} \tau=-\alpha_{2} e^{\rho-\tau} \xi_{1}, \operatorname{grad}^{2} \tau=-\beta_{2} \xi_{2}
\end{gathered}
$$

where $\alpha_{1}, \beta_{1}$ are functions on $M_{1}$ and $\alpha_{2}, \beta_{2}$ are functions on $M_{2}$. If $M_{1}$ and $M_{2}$ are trans-Sasakian then it is seen directly that all the components of (5.1)-(5.4) vanish, giving the following result.

Proposition 5.3. If one of the following three conditions is satisfied, the other two are equivalent:

(a) $\left(M_{1} \times M_{2}, J, \tilde{g}\right)$ is Kaehlerian

(b) The structures on $M_{1}$ and $M_{2}$ are trans-Sasakian (of types $\left(\alpha_{1}, \beta_{1}\right)$ and $\left(\alpha_{2}, \beta_{2}\right)$ respectively $)$

(c) $\operatorname{grad}^{1} \rho=-\beta_{1} \xi, \operatorname{grad}^{2} \rho=\alpha_{1} e^{\tau-\rho} \xi_{2}, \operatorname{grad}^{1} \tau=-\alpha_{2} e^{\rho-\tau} \xi_{1}, \operatorname{grad}^{2} \tau=-\beta_{2} \xi_{2}$.

We now turn to our main result.

Theorem. Let $M_{1}$ and $M_{2}$ be almost contact metric manifolds and $U$ a coordinate neighborhood on $M_{2}$ such that $\xi_{2}=\frac{\partial}{\partial t}$. Consider the change of metric $\tilde{g}=e^{2 \rho} g_{1}+e^{2 \tau} g_{2}$ on $M_{1} \times U$ given $b y$

$$
\rho=\ell n\left(k-\frac{\alpha}{\beta} e^{-\beta t}\right), r=-\beta t
$$

where $\alpha \neq 0, \beta \neq 0$ and $k$ are constants such that $\rho$ is defined on $U$. Then $\left(M_{1} \times U, J, \tilde{g}\right)$ is Kaehlerian if and only if the structure on $M_{1}$ is $\alpha$-Sasakian and the structure on $U$ is a $\beta-K$ enmotsu.

Proof: First note that $\xi_{2} \rho=\alpha e^{\tau-\rho}$ and $\xi_{2} \tau=-\beta$. Now suppose that the structure on $M_{1} \times U$ is Kaehlerian. Then from the first component of (5.1) we see that $M_{1}$ is $\alpha$-Sasakian. Now in the first component of (5.3) choose $Y_{2}$ orthogonal to $\xi_{2}$; then $\left(\phi_{2} Y_{2} \rho\right) X_{1}-\left(Y_{2} \rho\right) \phi_{1} X_{1}=0$ from which we have $Y_{2} \rho=0$. Therefore

$$
0=\frac{\alpha e^{-\beta t}}{e^{\rho}}\left(Y_{2} t\right)
$$

giving $Y_{2} t=0$ and hence $Y_{2} \tau=0$. Thus

$$
\operatorname{grad}^{2} \tau=-\beta \xi_{2} .
$$


Now using the second component of (5.2) we have

$$
\left(\nabla^{2} X_{2} \phi_{2}\right) Y_{2}=\beta\left(g_{2}\left(\phi_{2} X_{2}, Y_{2}\right) \xi_{2}-\eta_{2}\left(Y_{2}\right) \phi_{2} X_{2}\right),
$$

i.e. the structure on $U$ is $\beta$-Kenmotsu.

Converseiy since the structure on $U$ is $\beta$-Kenmotsu, $\nabla_{X_{2}}^{2} \xi_{2}=\beta\left(X_{2}-\eta_{2}\left(X_{2}\right) \xi_{2}\right)$ from which $d \eta_{2}=0$. Thus the subbundle $\eta_{2}=0$ is integrable. Therefore $Y_{2} t=0$ for any vector field $Y_{2}$ orthogonal to $\xi_{2}$ and hence

$$
\operatorname{grad}^{2} \tau=-\beta \xi_{2}
$$

and

$$
\operatorname{grad}^{2} \rho=\alpha e^{\tau-\rho} \xi_{2} .
$$

Moreover $\operatorname{grad}^{1} \rho=\operatorname{grad}^{1} \tau=0$. Now, by using Proposition 5.3 we see that $\left(M_{1} \times U, J, \tilde{g}\right)$ is Kaehlerian.

Remarks. 1. The conformal change $\rho=\tau=t_{2}$ gives $M_{1}$ Sasakian and $U(-1)-$ Kenmotsu which means that $\left(\phi_{2},-\xi_{2},-\eta_{2}, g_{2}\right)$ is a Kenmotsu structure. The choice $\rho=\ell n\left(k-e^{-t_{2}}\right), \tau=-t_{2}$ gives $M_{1}$ Sasakian and $U$ Kenmotst directly.

2. The fact that the theorem is local in regard to the second manifold $M_{2}$ is not unnatural. Even for $M_{1} \times \mathbf{R}$, the 1-dimensional case for $M_{2}$, note that the Hopf manifold $S^{2 n+1} \times S^{1}$ is locally conformaily Kaehier but not globally conformally Kaehler.

\section{References}

1. BlaIR, D.E., "Contact Manifolds in Riemannian Geometry," Lecture Notes in Mathematics 509, Springer, 1976.

2. CAPURSI, M., Some remarks on the product of two almost contact manifolds, An. Sti. Univ. "Al. I. Cuza" XXX (1984), 75-79.

3. Gray A. AND Hervelia, L.M., The sixteen classes of almost Hermitian manifolds and their linear invariants, Ann. Math. Pura Appl., (4) 123 (1980), 35-58.

4. IANUS, S. AND SMARANDA, D., Some remarkable structures on the product of an almost contact metric manifold with the real line, Papers from the National Colloquium on Geometry and Topology, Univ. Timisoara (1977), 107-110.

5. Janssens, D. AND VANHECKE, L., Almost contact structures and curvature tensors, Kōdai Math. J. 4 (1981), 1-27.

6. Kenmotsu, K., A class of almost contact Riemannian manifolds, Tohoku Math. J. 24 (1972), 93-103. 
7. Markeko, J.C., The local structure of trans-Sasakian manifolds (to appear).

8. Morimoto, A., On normal almost contact structures, J. Math. Soc. Japan 15 (1963), 420-436.

9. OUbiña, J.A., New classes of almost contact metric structures, Publicationes Mathematicae Debrecen 32 (1985), 187-193.

10. TANNo, S., The automorphism groups of almost contact Riemannian manifolds, Tôhoku Math. J. 21 (1969), 21-38.

D.E. Blair: Department of Mathematics Michigan State University

East Lansing, Michigan 48824

U.S.A.

J.A. Oubiña : Departamento de Geometria y Topologia

Facultad de Matemáticas

Universidad de Santiago de Compostela

Santiago de Compostela

SPAIN

Rebut el 7 de Jutiol de 1989 